\title{
Limited Predictive Value of FDG-PET for Response Assessment in the Preoperative Treatment of Esophageal Cancer: Results of a Prospective Multi-Center Trial (SAKK 75/02)
}

\author{
Bernd Klaeser ${ }^{a, b}$ Egbert Nitzsche ${ }^{c}$ Jan C. Schuller ${ }^{d}$ Dieter Köberle ${ }^{e} \quad$ Lucas Widmer $^{f}$ \\ Sabine Balmer-Majno ${ }^{g}$ Thomas Hany ${ }^{h}$ Corinne Cescato-Wenger ${ }^{i}$ Peter Brauchlid $^{d}$ \\ Michael Zünd ${ }^{k} \quad$ Bernhard C. Pestalozzil Clemens Caspar ${ }^{m} \quad$ Susanne Albrecht ${ }^{n}$ \\ Roger von Moos $^{\circ}$ Thomas Ruhstaller
}

\begin{abstract}
${ }^{a}$ Department of Nuclear Medicine, Inselspital Bern, ${ }^{\mathrm{b}}$ Department of Nuclear Medicine, Kantonsspital St. Gallen, ${ }^{\mathrm{c}}$ Department of Nuclear Medicine, Kantonsspital Aarau, ${ }^{d}$ Coordinating Center SAKK, Bern, ${ }^{e}$ Department of Oncology and Hematology, Kantonsspital St. Gallen, ${ }^{\mathrm{f}}$ Department of Oncology, Triemlispital Zurich, ${ }^{9}$ Department of Radiation Oncology, University Hospital of Geneva, ${ }^{\mathrm{h}}$ Department of Nuclear Medicine, University Hospital Zurich, ' Department of Medical Oncology, Claraspital Basel, ${ }^{\mathrm{k}}$ Department of Surgery, Kantonsspital St. Gallen, ' Department of Oncology, University Hospital Zurich, ${ }^{m}$ Department of Oncology and Hematology, Kantonsspital Baden, ${ }^{n}$ Department of Nuclear Medicine, University Hospital of Geneva, ${ }^{\circ}$ Department of Medical Oncology and Hematology, Kantonsspital Graubünden, Chur, Switzerland
\end{abstract}

\section{Key Words}

Esophageal cancer . Positron emission tomography (PET) .

Predictive marker · Response assessment

\section{Summary}

Background: Only responding patients benefit from preoperative therapy for locally advanced esophageal carcinoma. Early detection of non-responders may avoid futile treatment and delayed surgery. Patients and Methods: In a multi-center phase II trial, patients with resectable, locally advanced esophageal carcinoma were treated with 2 cycles of induction chemotherapy followed by chemoradiotherapy (CRT) and surgery. Positron emission tomography with 2[fluorine-18]fluoro-2-deoxy-D-glucose (FDG-PET) was performed at baseline and after induction chemotherapy. The metabolic response was correlated with tumor regression grade (TRG). A decrease in FDG tumor uptake of less than $40 \%$ was prospectively hypothesized as a predictor for histopathological non-response (TRG > 2) after CRT. Results: 45 patients were included. The median decrease in FDG tumor uptake after chemotherapy correlated well with TRG after completion of CRT ( $p=0.021)$. For an individual patient, less than $40 \%$ decrease in FDG tumor uptake after induction chemotherapy predicted histopathological non-response after completion of CRT, with a sensitivity of $68 \%$ and a specificity of $52 \%$ (positive predictive value 58\%, negative predictive value $63 \%$ ). Conclusions: Metabolic response correlated with histopathology after preoperative therapy. However, FDG-PET did not predict non-response after induction chemotherapy with sufficient clinical accuracy to justify withdrawal of subsequent CRT and selection of patients to proceed directly to surgery.

\section{Schlüsselwörter}

Ösophaguskarzinom - Positronenemissionstomografie (PET) . Marker, prädiktiver · Response-Vorhersage

\section{Zusammenfassung}

Hintergrund: Die präoperative Chemo- und Radiotherapie des lokal fortgeschrittenen Ösophaguskarzinoms ist nur für Patienten von Vorteil, die auf diese ansprechen. Durch eine frühe Identifikation von Patienten, die kein ausreichendes Ansprechen zeigen, könnten zwecklose Therapien und Verzögerungen der Operation vermieden werden. Patienten und Methoden: Patienten mit resektablem, lokal fortgeschrittenem Ösophaguskarzinom wurden in einer multizentrischen Phase-II-Studie mit 2 Zyklen Induktionschemotherapie behandelt, gefolgt von einer Chemoradiotherapie (CRT) und einer Operation. Eine Positronenemissionstomografie mit 2-[ $\left.{ }^{18} \mathrm{~F}\right]$ Fluor-2desoxy-D-glukose (FDG-PET) wurde vor Therapiebeginn und nach Abschluss der Induktionschemotherapie durchgeführt. Das metabolische Ansprechen wurde mit dem Tumorregressionsgrad (TRG) korreliert. Die Hypothese, dass weniger als 40\% Abnahme des Wertes der FDG-Aufnahme ein früher prädiktiver Parameter für das Nichtansprechen sei (TRG > 2), wurde prospektiv geprüft. Ergebnisse: $45 \mathrm{~Pa}$ tienten wurden eingeschlossen. Die mittlere Abnahme der FDG-Anreicherung im Tumor korrelierte gut mit dem TRG nach Abschluss der CRT $(p=0,021)$. Eine Abnahme der FDG-Anreicherung von weniger als $40 \%$ sagte ein Nichtansprechen mit einer Sensitivität von $68 \%$ und einer Spezifität von $52 \%$ voraus (positiver Vorhersagewert $58 \%$, negativer Vorhersagewert 63\%). Schlussfolgerungen: Das metabolische Ansprechen nach präoperativer Therapie korrelierte insgesamt gut mit dem histopathologischen Ansprechen. Die FDG-PET sagte jedoch das Nichtansprechen im individuellen Fall nicht mit ausreichender Sicherheit voraus, um den vorzeitigen Abbruch der CRT und eine direkte Operation zu rechtfertigen.

\begin{tabular}{ll}
\hline KARGER & $\oplus$ 2009 S. Karger GmbH, Freiburg \\
Fax +497614520714 & Accessible online at: \\
$\begin{array}{l}\text { Information@Karger.de } \\
\text { www.karger.com }\end{array}$ & www.karger.com/onk
\end{tabular}

\section{KARGER}

Bernd Klaeser, MD

Department of Nuclear Medicine, Inselspital

Bern University Hospital and University of Bern

CH-3010 Bern, Switzerland

Tel. +41 632-2656, Fax -3137

bernd.klaeser@insel.ch 


\section{Introduction}

Esophageal carcinoma is often diagnosed in the locally advanced stage and is associated with a poor long-term outcome of only $20-30 \%$ survival at 2 years. Chemoradiotherapy (CRT) followed by surgery has been integrated into standard treatment. However, only patients who respond to neoadjuvant therapy and achieve an $\mathrm{R} 0$ resection at surgery have a substantial long-term survival, while non-responders may not benefit [1] but experience adverse effects or even tumor progression. In this context, early differentiation of non-responders from responders is desirable to prevent non-responders from receiving inefficient chemotherapy and delayed surgery. Positron emission tomography with 2-[fluorine-18]fluoro-2deoxy-D-glucose (FDG-PET) visualizes the enhanced glucose metabolism of viable esophageal tumor tissue $[2,3]$. A number of studies have addressed the feasibility of measuring changes in tumor FDG uptake to monitor response and to predict the outcome of neoadjuvant treatment.

Promising results for early response assessment after neoadjuvant chemotherapy without concomitant radiotherapy were published for adenocarcinomas of the esophagogastric junction [4]. Metabolic response was defined by a retrospectively calculated cut-off of least $35 \%$ decrease in tumor FDG uptake after 14 days of chemotherapy. Metabolic response correlated significantly with clinical and histopathological response, disease-free survival and overall survival (OS). A subsequent study by the same group prospectively tested this cut-off and confirmed that metabolic non-responders had a low histopathological response rate of only $5 \%$ and a poor prognosis compared with responders [5].

In contrast, single-center studies of CRT for adenocarcinoma and/or squamous cell carcinoma (SCC), designed to assess the correlation between a decrease in tumor FDG uptake and histopathological response [6-15] and survival [6, 7, 9, 13, 15], have produced conflicting results. A group from Munich, Germany, evaluated PET for the response assessment in the course of CRT of SCC. They found PET, after completion of CRT and early in the course of CRT, to have similar predictive values for histopathological response $[9,15]$.

Subsequently, the same group prospectively tested a predefined cut-off of $30 \%$ decrease in tumor standardized uptake values (SUV) after 2 weeks of CRT. However, the negative predictive value (NPV) for histopathological response remained clearly below $80 \%$ and, consequently, did not meet the requirement for a clinically relevant predictive test [16]. Radiation-induced esophagitis, which can mask treatment-induced reduction of SUV, may confound metabolic response evaluation after CRT [6, 8, 10, 11, 15].

This study was designed to quantify metabolic tumor response without interference from radiation-induced esophagitis, by determining whether changes in tumor FDG uptake after induction chemotherapy, but prior to CRT, can predict histopathological non-response after completion of neoadju- vant treatment. A cut-off of $40 \%$ SUV decrease for the differentiation of non-responders from responders was prospectively defined, based on data from previous studies [4, 9]. The primary objective was to predict histopathological non-response. Secondary objectives were to correlate metabolic response with event-free survival (EFS) and OS and to determine whether metabolic response may be a useful prognostic parameter.

\section{Patients and Methods}

\section{Patients}

PET imaging was performed as part of a prospective multi-center phase II trial investigating a cisplatin- and taxane-based regimen of 2 cycles of induction chemotherapy, followed by CRT and surgery (Swiss Group for Clinical Cancer Research, SAKK 75/02, NCT00072033) [17]. The protocol was approved by the local ethics committees of the participating centers. The study population consisted of previously untreated patients with histologically confirmed locally advanced but resectable SCC and adenocarcinoma of the thoracic esophagus or esophagogastric junction classified as clinical stage T3 N0, T1-3 N+ or T4 Nx, according to the AJCC Cancer Staging Manual, 6th edition [18]. Written informed consent was obtained for all patients. Contrast-enhanced computed tomography (CT) of the thorax and abdomen and endoluminal ultrasound of the esophagus were performed in all patients before PET or PET-CT. PET or PET-CT staging was strongly recommended in the study protocol, but was not mandatory because PET scanners were not available in all regions of Switzerland. Patients with distant metastases detected during pre-treatment evaluation were excluded.

\section{PET Imaging}

PET or PET-CT was performed at up to 4 weeks before initiation of induction chemotherapy and after completion of 2 cycles of induction chemotherapy in week 5, before the start of CRT (fig. 1). Both PET scans were performed at the same center on the same PET or PET-CT machine and under identical conditions for each patient. All PET scanners fulfilled the quality requirements defined by the Swiss Society of Nuclear Medicine and had a spatial resolution of $6 \mathrm{~mm}$ or less.

Patients fasted for at least $6 \mathrm{~h}$ before an intravenous injection of 5 $\mathrm{MBq}$ FDG/kg bodyweight. The blood glucose level was recorded in all patients. Patients were examined according to the local acquisition protocols at each center, and acquisition parameters were kept constant for both PET scans with regard to the time point of acquisition after tracer injection. Maximum SUV ( $\mathrm{SUV}_{\max }$ ) of the primary tumor was calculated to semi-quantify FDG tumor uptake. Percentage changes of SUV $\mathrm{max}_{\text {be- }}$ tween baseline PET and PET after induction chemotherapy were calculated to quantify metabolic response. PET data were evaluated at each site and reviewed centrally.

\section{Preoperative Treatment}

The preoperative treatment regimen consisted of induction chemotherapy with intravenous cisplatin $75 \mathrm{mg} / \mathrm{m}^{2}$ and docetaxel $75 \mathrm{mg} / \mathrm{m}^{2}$ on days 1 and 21, followed by radiotherapy (total dose $45 \mathrm{~Gy}$ ) and concurrent chemotherapy comprising intravenous cisplatin $25 \mathrm{mg} / \mathrm{m}^{2}$ and docetaxel $20 \mathrm{mg} / \mathrm{m}^{2}$ weekly for 5 weeks. Surgery was scheduled 3 to 8 weeks after CRT (fig. 1). Patients with evidence of newly detected stage M1 and/or inoperable T4 disease were not eligible for surgery.

\section{Criteria for Response}

Histopathological response was based on pathological findings after esophagectomy. Specimens were examined according to standardized procedures in local pathology laboratories, and all specimens were centrally reviewed at the University of Basel by an experienced pathologist. 


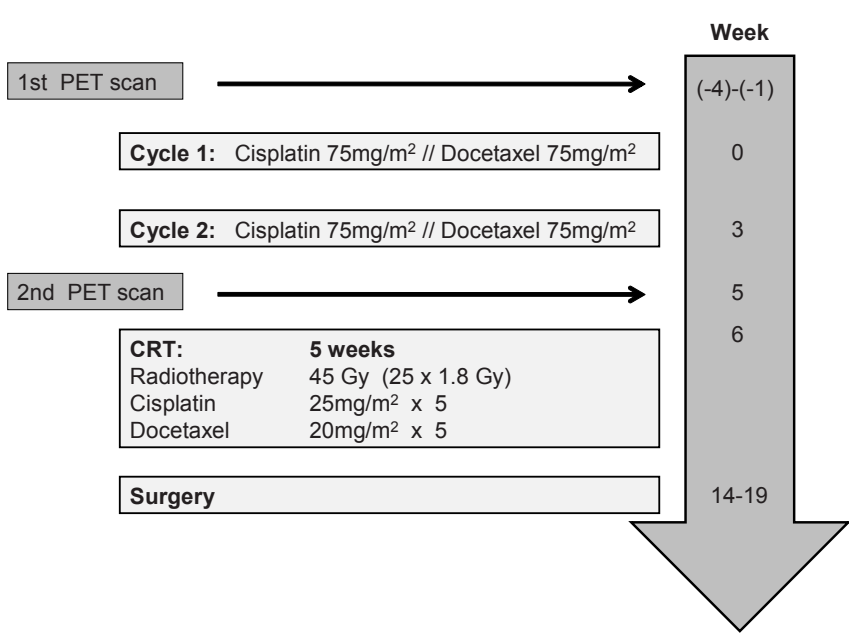

Fig. 1. Study schedule: 1st PET scan before chemotherapy, 2nd PET scan in the last week before initiation of CRT.

The histopathological response was classified according to the Mandard classification of tumor regression grade (TRG) [19]. TRG 1 was defined as complete regression, TRG 2 as presence of rare residual cancer cells scattered throughout the fibrosis, and TRG $>2$ as increased number of residual cancer cells. Patients with complete regression (TRG 1) or nearcomplete regression (TRG 2) were classified as responders, while patients with partial and minimal response and no change (TRG 3-5) were classified as non-responders, as previously validated [15]

A cut-off of $40 \%$ decrease in tumor FDG uptake between initial and second FDG-PET scan was prospectively hypothesized to discriminate non-responders from responders, based on previously published optimal cut-off values for response assessment after chemotherapy and CRT of adenocarcinoma and SCC $[4,9]$. More than $40 \%$ decrease in FDG uptake from baseline to the second chemotherapy cycle was considered as a metabolic response to chemotherapy.

\section{Statistical Methods}

The sensitivity, positive predictive value (PPV), specificity, and negative predictive value (NPV) of metabolic non-response (i.e. less than $40 \%$ decrease in FDG uptake) predicting TRG $>2$ was calculated. Survival endpoints and $95 \%$ confidence intervals (CIs) were calculated using the KaplanMeier method. The survival times of strata (metabolic responders vs. nonresponders, adenocarcinoma vs. SCC, histopathological responders vs. non-responders) were compared with the log-rank test. Overall survival was calculated as the time from registration until death (event) or last follow-up (censored). EFS was calculated as time from registration until death or progression (event) or last follow-up (censored). The reverse Kaplan-Meier method was used to calculate the median follow-up time. The difference of means or medians was tested with the $t$-test or Wilcoxon's test. Calculations and plots were performed with SAS 9.1 and S-Plus 7.0

\section{Results}

\section{Patient and Tumor Characteristics}

Of 66 patients enrolled in the SAKK 75/02 trial, 57 underwent surgical tumor resection and assessment of histopathological response. From this population, 45 patients were included in the PET study. The remaining 12 patients were not included for the following reasons: baseline PET scans not performed ( 9 patients; 6 because study centers did not participate in the

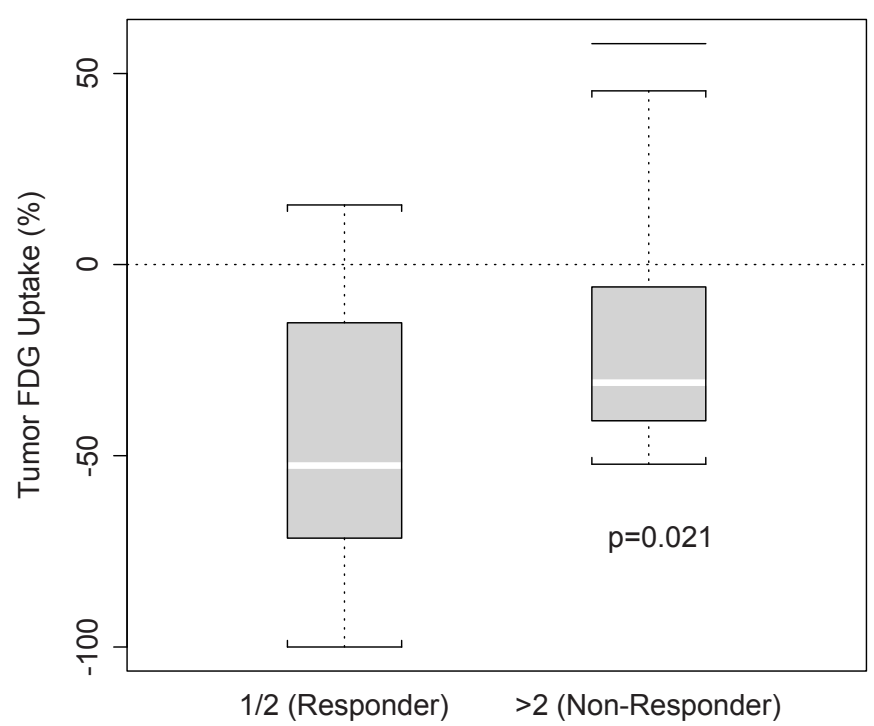

Fig. 2. Decrease in tumor FDG uptake after chemotherapy in histopathological responders vs. non-responders (white line: median, box: 1st and 3 rd quartile). Horizontal dotted reference line: no change in tumor FDG uptake. Wilcoxon's test showed a significant difference $(\mathrm{p}=0.021)$ in relative change of tumor FDG uptake between histopathological responders (TRG 1/2) and non-responders (TRG > 2).

PET study, 1 patient refused, and 2 scans were not correctly scheduled), 2 patients could not be evaluated because the primary tumor did not show an increased FDG uptake in the baseline scan, and 1 patient did not have the second PET.

The median age was 61 years for patients with adenocarcinoma (range 48-71 years) and 59 years for those with SCC (range 44-70 years). The patient characteristics are listed in table 1.

\section{Change of Tumor FDG Uptake after Two Cycles of \\ Chemotherapy}

The median relative change in FDG uptake after induction chemotherapy compared with baseline uptake was $-53 \%$ for patients with histological complete or near-complete response (TRG $1 / 2$ ) and $-31 \%$ for non-responders with an increased number of residual cancer cells (TRG $>2)$. This difference was statistically significant (Wilcoxon's test: $p=0.021$; fig. 2).

\section{Prediction of Histopathological Non-Response after CRT}

The prospectively defined cut-off value of less than $40 \%$ decrease in FDG uptake after 2 cycles of induction chemotherapy did not reliably predict pathological non-response after completion of CRT (fig. 3). The overall sensitivity and specificity for prediction of non-response were 68 and 52\%, respectively, resulting in a PPV of $58 \%$ and an NPV of $63 \%$. The sensitivity, specificity, PPV and NPV for non-response were $60,50,64$ and $45 \%$ for adenocarcinoma and 86, 54, 50 and $88 \%$ for SCC, respectively. Moreover, no significant differences in the prediction of non-response according to SUV decrease were found between patients presenting with adenocarcinoma or SCC. 
Table 1. Patient and tumor characteristics

Adenocarcinoma $(\mathrm{n}=25) \quad$ Squamous cell carcinoma $(\mathrm{n}=20) \quad$ All $(\mathrm{n}=45)$

\begin{tabular}{|c|c|c|c|c|}
\hline \multicolumn{5}{|l|}{ Age, years } \\
\hline Median & 61 & 59 & 60 & \\
\hline Range & $48-71$ & $44-70$ & $44-7$ & \\
\hline \multirow[t]{2}{*}{ Median follow-up, months } & 27 & 30 & 28 & \\
\hline & $\mathrm{n}$ & $\mathrm{n}$ & $\mathrm{n}$ & $\%$ \\
\hline \multicolumn{5}{|l|}{ Sex } \\
\hline Male & 23 & 17 & 40 & 89 \\
\hline Female & 2 & 3 & 5 & 11 \\
\hline \multicolumn{5}{|l|}{ Clinical stage at diagnosis } \\
\hline T1 N1 & 0 & 0 & 0 & 0 \\
\hline $\mathrm{T} 2 \mathrm{~N} 1$ & 5 & 3 & 8 & 18 \\
\hline T3 N0 & 4 & 2 & 6 & 13 \\
\hline T3 N1 & 15 & 13 & 28 & 62 \\
\hline $\mathrm{T} 3 \mathrm{Nx}$ & 1 & 2 & 3 & 7 \\
\hline T4 N1 & 0 & 0 & 0 & 0 \\
\hline \multicolumn{5}{|l|}{ WHO tumor grading } \\
\hline 1 & 2 & 3 & 5 & 11 \\
\hline 2 & 11 & 12 & 23 & 51 \\
\hline 3 & 10 & 5 & 15 & 33 \\
\hline Not done & 2 & 0 & 2 & 5 \\
\hline \multicolumn{5}{|c|}{ Dysphagia grade at diagnosis (NCI-CTC grading v2.0) } \\
\hline $0 / 1$ & 18 & 12 & 30 & 67 \\
\hline 2 & 6 & 7 & 13 & 29 \\
\hline 3 & 0 & 1 & 1 & 2 \\
\hline 4 & 1 & 0 & 1 & 2 \\
\hline \multicolumn{5}{|l|}{ Tumor regression grade } \\
\hline TRG 1 (pCR) & 2 & 5 & 7 & 16 \\
\hline TRG 2 & 8 & 8 & 16 & 35 \\
\hline TRG 3-5 & 15 & 7 & 22 & 49 \\
\hline
\end{tabular}

WHO, World Health Organization; NCI-CTC, National Cancer Institute common toxicity criteria; TRG, tumor regression grade; pCR, pathological complete response.

Figure 4 shows retrospectively calculated PPV for different cut-off values for tumor SUV decrease after chemotherapy. Of note, only an increase in FDG uptake would provide a markedly better PPV than the predefined cut-off of less than $40 \%$ decrease. However, this accounted for only 5 patients. In addition, 11 patients classified as metabolic non-responders after induction chemotherapy (5 adenocarcinoma, 6 SCC, patients originating from different participating centers) had histological complete or near-complete response after completion of CRT.

Prognostic Significance of Metabolic Response to Treatment Median follow-up was 28 months (adenocarcinoma 27 months, SCC 30 months). Median OS was 32.4 months (95\% CI lower limit 25.7 months). Metabolic responders according to the predefined criterion of at least $40 \%$ decrease in tumor FDG uptake had a median OS of 36.5 months (95\% CI 8.0-36.5 months) compared with 31.0 months (95\% CI lower limit 21.4 months) for non-responders. Median EFS was 22.8 months
(95\% CI lower limit 7.3 months) for metabolic responders and 21.7 months (95\% CI 9.8-28.3 months) for non-responders. Differences in OS and EFS failed to reach significance (log-rank test).

\section{Discussion}

To date, the utility of FDG-PET for early response assessment has been shown only after neoadjuvant chemotherapy alone. Promising results using retrospectively calculated or prospectively defined cut-off values have been published based on extensive work at a single center [4, 5, 20-22], and prospective multi-center trials are planned [23]. However, a role for FDG-PET in the response assessment after combined CRT has not been established and published single-center studies have shown conflicting results. While some studies suggested a possible correlation between the decrease in FDG tumor uptake and histopathological response [7-9, 15], these results 


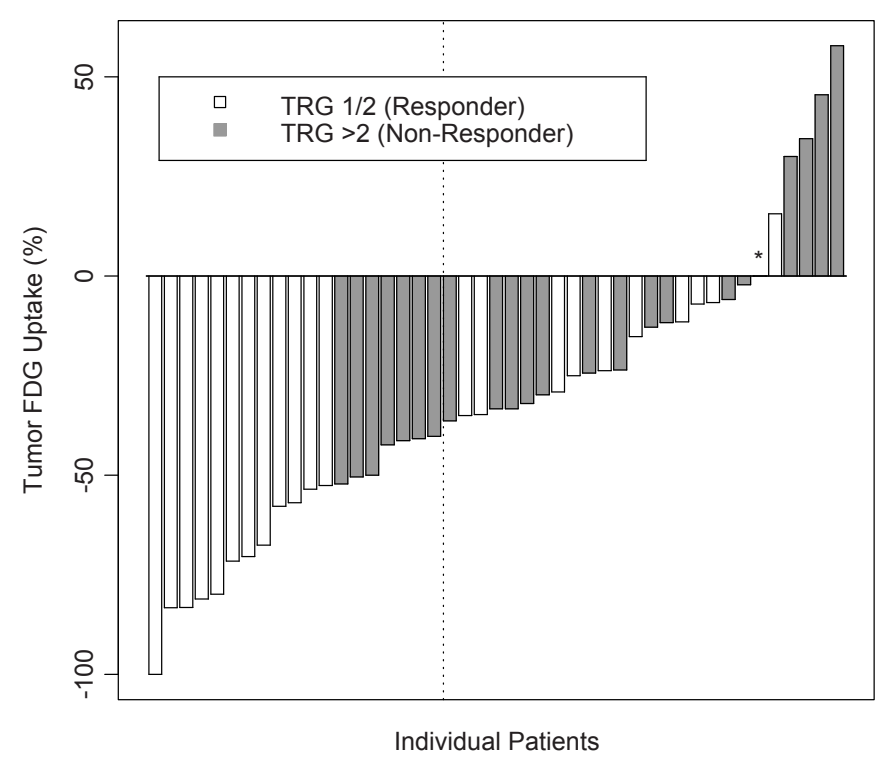

Fig. 3. 'Waterfall' plot: Outcome of patients with at least $40 \%$ reduction in SUV after chemotherapy (dotted line). One patient had no change in SUV; thus, the 6th bar from the right (a histopathological responder, marked with *) has a height of zero.

were not supported for adenocarcinoma or SCC by other, subsequent trials [10-14, 16]. Radiation-induced esophagitis correlates metabolically with FDG-PET in up to $60 \%$ of patients after radiotherapy and may explain obvious differences in response assessment after chemotherapy and CRT $[6,8,10$, $11,15]$.

The patients in the current study were treated with preoperative induction chemotherapy followed by CRT. It is critical to identify non-responders to neoadjuvant treatment so that they can be scheduled for immediate surgery. No optimal cutoff values for response assessment in this setting were available. Therefore, based on previously published studies with optimal cut-off values for response assessment after chemotherapy alone (35\%, adenocarcinoma [4]) and after completion of combined CRT (52\%, SCC [9]), it was hypothesized that a prospectively defined cut-off of less than $40 \%$ decrease in tumor FDG would identify non-responding adenocarcinoma and/or SCC after completion of induction chemotherapy.

For the whole group, the median change of FDG tumor uptake after 2 cycles of induction chemotherapy correlated significantly with the TRG after completion of CRT ( $\mathrm{p}=$ 0.021 ). These results were in accordance with those published for response assessment after chemotherapy alone $[4,5]$ but without chemoradiation before surgery. After a median follow-up of 28 months there was a trend towards prolonged OS for metabolic responders vs. non-responders after induction chemotherapy. No differences in EFS were found between metabolic responders and non-responders. Therefore, these results did not confirm previously published single-center studies describing a significant correlation between metabolic response and EFS and/or OS [4, 6, 7, 9, 20].

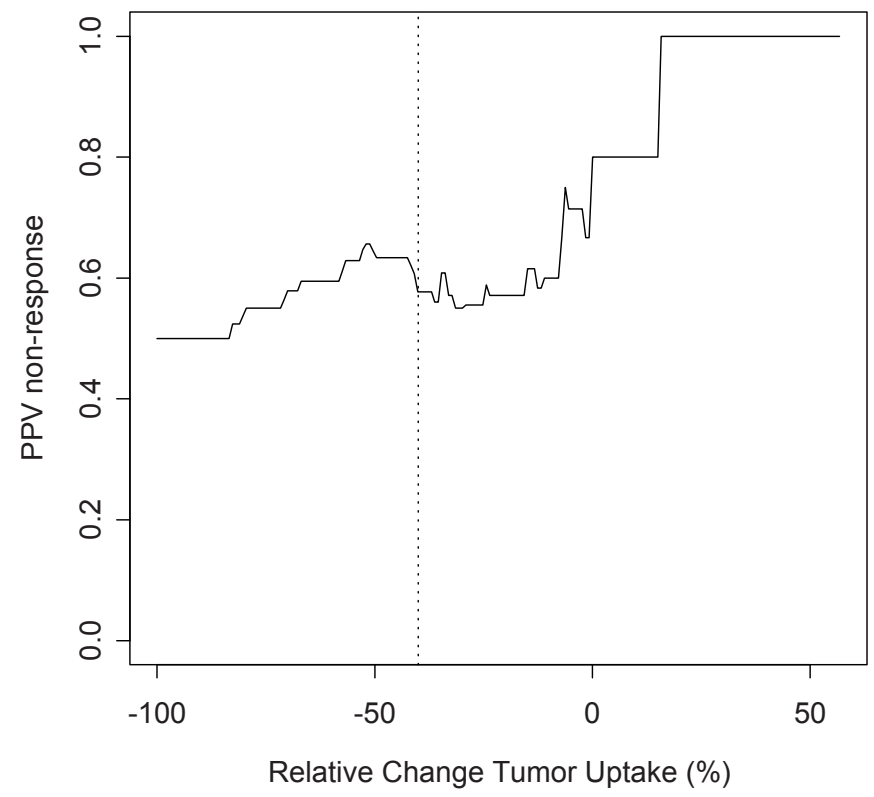

Fig. 4. $P P V$ for non-response of different cut-off values for relative change of SUV after chemotherapy. Dotted line: predefined cut-off of $40 \%$ SUV decrease.

The hypothesis that FDG-PET with a predefined cutoff of less than $40 \%$ decrease in tumor SUV after induction chemotherapy may predict histological non-response and treatment failure was not proven. The PPV for prediction of non-response of $58 \%$ was not sufficiently accurate to justify withdrawal of CRT in metabolic non-responders and to select patients to proceed directly to surgery. A retrospective calculation of an optimal cut-off did not improve the results (fig. 4) because only an increase in SUV reliably pointed towards non-response. These results were less favorable than those of other studies of adenocarcinoma in which the second PET was performed 2 weeks after initiation of chemotherapy alone (PPV for non-response, 95-100\%) [4, 5]. Lordick et al. [20] noted no histological responders among metabolic non-responders (less than $35 \%$ SUV decrease; response assessment 2 weeks after initiation of chemotherapy), while in the present study 11 of 26 metabolic non-responders (5 adenocarcinoma, 6 SCC, less than $40 \%$ SUV decrease) had histological complete or near-complete response after completion of CRT (specificity $52 \%$; fig. 3). Apparently, subsequent CRT can transform some metabolic non-responders after chemotherapy into histopathological responders. These 11 metabolic non-responders after induction chemotherapy with subsequent histopathological response (TRG 1/2) after completion of CRT had median EFS similar to all patients with histopathological response. It can be concluded that histopathological response was a better predictor of outcome than metabolic response in this study. Brücher et al. reported promising results for SCC evaluated 3 weeks after completion of CRT (PPV for non-response 100\%, with a retrospectively defined cut-off of 52\%) [9]. However, in a follow-up study by the same group, using a prospectively 
defined cut-off of $30 \%$, the PPV for non-response (64\%) did not meet the requirement for a clinically relevant predictive test [16] and was in the same range as data from Song et al. [8] using a second PET performed 8 weeks after CRT.

To the authors' knowledge, this is the first study prospectively testing the predictive and prognostic value of FDG-PET in esophageal cancer in a nationwide multi-center context. A strong point of this study is the assessment of non-response as the objective of clinical relevance using a prospectively defined cut-off of tumor SUV decrease during the course of treatment. A new approach using a second PET after induction chemotherapy and before the onset of combined CRT was evaluated to avoid problems with semi-quantification of tumor activity, i.e. radiation-induced esophagitis [10]. A limitation of this study is the small number of patients and maybe the combined evaluation of adenocarcinoma and SCC. Recently, separate trials for adenocarcinoma and SCC have been proposed because the thresholds for measuring PET response may vary [23]. However, all patients in the study received identical treatment and no significant differences in the prediction of non-response according to SUV decrease were found between adenocarcinoma and SCC. Using a multi-center design means that the mode of acquisition and the instrumentation were standardized to a lesser degree than would be possible in a single-center study. Nevertheless, only relative changes of FDG uptake in the pre- and post-treatment scans were evaluated, and all acquisition parameters were kept uniform in individual patients. Under such conditions, no significant effects of methodological variations, i.e. acquisition parameters, timing of the acquisition after FDG injection, reconstruction algorithm and method of SUV measurement, have been described to date $[13,23-25]$, indicating that the results of this trial can be regarded as reliable. Consensus recommendations for the use of FDG-PET as an indicator of therapeutic response in multi-center trials, which have been published in the meantime [26], had already been essentially respected in the design of this trial.

In conclusion, metabolic response after induction chemotherapy correlated well with histopathological response after completion of CRT. However, response assessment with FDGPET using semi-quantitative uptake measurement was not able to predict histological non-response after neoadjuvant treatment with sufficient clinical accuracy to select patients to proceed directly to surgery. It remains to be determined whether a more reliable response prediction may be possible by employing recent technical developments including high-resolution PET-CT, partial volume and recovery correction, and kinetic modeling.

\section{Acknowledgements}

We thank Sandra Thierstein, trial coordinator of the SAKK Bern, for her tremendous administrative support for this trial, and Pierluigi Ballabeni, Ph.D., for assistance in designing this trial. The Swiss Group for Clinical Cancer Research (SAKK) was the sponsor of the trial and was financially supported by the Federal Government of Switzerland and Sanofi-Aventis Switzerland.

\section{Conflict of Interest Statement}

All authors: None declared.

\section{References}

1 Kelsen DP, Winter KA, Gunderson LL, et al.: Longterm results of RTOG trial 8911 (USA Intergroup 113): a random assignment trial comparison of chemotherapy followed by surgery compared with surgery alone for esophageal cancer. J Clin Oncol 2007; 25:3719-3725.

2 Flanagan FL, Dehdashti F, Siegel BA, et al.: Staging of esophageal cancer with $18 \mathrm{~F}$-fluorodeoxyglucose positron emission tomography. AJR Am J Roentgenol 1997;168:417-424.

3 Flamen P, Lerut A, Van Cutsem E, et al.: Utility of positron emission tomography for the staging of patients with potentially operable esophageal carcinoma. J Clin Oncol 2000;18:3202-3210.

$\checkmark 4$ Weber WA, Ott K, Becker K, et al.: Prediction of response to preoperative chemotherapy in adenocarcinomas of the esophagogastric junction by metabolic imaging. J Clin Oncol 2001;19:3058-3065.

$\checkmark 5$ Ott K, Weber WA, Lordick F, et al.: Metabolic imaging predicts response, survival, and recurrence in adenocarcinomas of the esophagogastric junction. $\mathrm{J}$ Clin Oncol 2006;24:4692-4698.

6 Flamen P, Van Cutsem E, Lerut A, et al.: Positron emission tomography for assessment of the response to induction radiochemotherapy in locally advanced oesophageal cancer. Ann Oncol 2002;13:361-368.
7 Swisher SG, Maish M, Erasmus JJ, et al.: Utility of PET, CT, and EUS to identify pathologic responders in esophageal cancer. Ann Thorac Surg 2004; 78:1152-1160.

8 Song S, Kim JH, Ryu JS, et al.: FDG-PET in the prediction of pathologic response after neoadjuvant chemoradiotherapy in locally advanced, resectable esophageal cancer. Int J Radiat Oncol Biol Phys 2005;63:1053-1059.

9 Brücher BL, Weber W, Bauer M, et al.: Neoadjuvant therapy of esophageal squamous cell carcinoma: response evaluation by positron emission tomography. Ann Surg 2001;233:300-309.

10 Brink I, Hentschel M, Bley TA, et al.: Effects of neoadjuvant radio-chemotherapy on 18F-FDG-PET in esophageal carcinoma. Eur J Surg Oncol 2004; 30:544-550.

11 Gillham CM, Lucey JA, Keogan M, et al.: (18)FDG uptake during induction chemoradiation for oesophageal cancer fails to predict histomorphological tumor response. Br J Cancer 2006;95:1174-1179.

12 Smithers BM, Couper GC, Thomas JM, et al.: Positron emission tomography and pathological evidence of response to neoadjuvant therapy in adenocarcinoma of the esophagus. Dis Esophagus 2008;21: 151-158.
13 Schmidt M, Bollschweiler E, Dietlein M, et al Mean and maximum standardized uptake values in [18F]FDG-PET for assessment of histopathological response in oesophageal squamous cell carcinoma or adenocarcinoma after radiochemotherapy. Eur J Nucl Med Mol Imaging 2009;36:735-744.

14 Konski AA, Cheng JD, Goldberg M, et al.: Correlation of molecular response as measured by 18 -FDG positron emission tomography with outcome after chemoradiotherapy in patients with esophageal carcinoma. Int J Radiat Oncol Biol Phys 2007;69:358-363.

15 Wieder HA, Brücher BL,Zimmermann F, et al.:Time course of tumor metabolic activity during chemoradiotherapy of esophageal squamous cell carcinoma and response to treatment. J Clin Oncol 2004;22: 900-908.

16 Brücher BL, Wieder HA, Busch R, et al.: Possible individualized therapy by early assessment of response during neoadjuvant radiochemotherapy in esophageal squamous cell carcinoma patients by 18 FDG-PET. J Clin Oncol 2006;24(suppl): abstr 4060.

17 Ruhstaller T, Widmer L, Schuller JC, et al.: Multicenter phase II trial of preoperative induction chemotherapy followed by chemoradiation with docetaxel and cisplatin for locally advanced esophageal carcinoma (SAKK 75/02). Ann Oncol 2009;20:1522-1528. 
18 Greene FL, Page DL, Fleming ID, et al. (eds): AJCC cancer staging manual, ed 6. New York, Springer, 2002.

19 Mandard AM, Dalibard F, Mandard JC, et al. Pathologic assessment of tumor regression after preoperative chemoradiotherapy of esophagea carcinoma. Clinicopathologic correlations. Cancer 1994;73:2680-2686.

20 Lordick F, Ott K, Krause BJ, et al.: PET to assess early metabolic response and to guide treatment of adenocarcinoma of the oesophagogastric junction: the MUNICON phase II trial. Lancet Oncol 2007;8:797-805.
Wieder HA, Beer AJ, Lordick F, et al.: Comparison of changes in tumor metabolic activity and tumor size during chemotherapy of adenocarcinomas of the esophagogastric junction. J Nucl Med 2005;46:2029-2034.

22 Wieder HA, Ott K, Lordick F, et al.: Prediction of tumor response by FDG-PET: comparison of the accuracy of single and sequential studies in patients with adenocarcinomas of the esophagogastric junction. Eur J Nucl Med Mol Imaging 2007;34:1925-1932.

23 Lordick F, Ruers T, Aust DE, et al.; European Organisation of Research and Treatment of Cancer Gastrointestinal Group: European Organisation of Research and Treatment of Cancer (EORTC) Gastrointestinal Group: Workshop on the role of metabolic imaging in the neoadjuvant treatment of gastrointestinal cancer. Eur J Cancer 2008;44:1807-1819.
24 Stahl A, Ott K, Schwaiger M, et al.: Comparison of different SUV-based methods for monitoring cytotoxic therapy with FDG PET. Eur J Nucl Med Mol Imaging 2004;31:1471-1478.

25 Souvatzoglou M, Ziegler SI, Martinez MJ, et al.: Standardised uptake values from PET/CT images: comparison with conventional attenuation-corrected PET. Eur J Nucl Med Mol Imaging 2007;34:405-412.

26 Shankar LK, Hoffman JM, Bacharach S, et al.: Consensus recommendations for the use of $18 \mathrm{~F}-\mathrm{FDG}$ PET as an indicator of therapeutic response in patients in National Cancer Institute trials. J Nucl Med 2006;47:1059-1066. 